\title{
TECNOLOGÍAS DE LA INFORMACIÓN PARA RESOLVER CONTINGENCIAS EN LA AFILIACIÓN AL RÉGIMEN SUBSIDIADO DE SALUD EN PERÚ: "RESUELVE TU AFILIACIÓN"
}

\author{
José Villegas-Ortega1,2,a, César Loyola-Martínez ${ }^{1, b}$, Javier Santisteban-Romero,c, \\ Mónica Manchego-Lombardi ${ }^{1, c}$, Michelle Lozada-Urbano ${ }^{1,3, \mathrm{~d}}$
}

\begin{abstract}
RESUMEN
La Superintendencia Nacional de Salud (SUSALUD) ha desarrollado la plataforma en línea: "ReSUelve tu afiliación" con el propósito de solucionar problemas de acceso a los servicios de salud de los ciudadanos peruanos que cuentan con un seguro de salud en las instituciones administradoras de fondos de aseguramiento en salud (IAFAS). Esta plataforma articula virtualmente a las principales IAFAS en Perú, las cuales reciben solicitudes de cualquier usuario que requiera actualización del estado de su filiación, para resolverlas en menos de $24 \mathrm{~h}$. En casi ocho meses de implementación se han resuelto más de 55 mil solicitudes, con lo cual se ha logrado que los usuarios accedan de forma oportuna a los servicios de salud bajo la cobertura correspondiente. De esta manera, se contribuye a garantizar el derecho al acceso a los servicios de salud, derecho que se veía vulnerado por los retrasos en los trámites de afiliación entre las diferentes IAFAS del país.
\end{abstract}

Palabras clave: Seguro de Salud; Accesibilidad a los servicios de salud; Sistemas de información; Cobertura de los servicios de salud; Perú (fuente: DeCS BIREME).

\section{INFORMATION TECHNOLOGIES INTENDED TO SOLVE CONTINGENCIES IN THE PERUVIAN SUBSIDIZED HEALTH SYSTEM AFFILIATION: "RESUELVE TU AFILIACIÓN"}

\begin{abstract}
The National Health Authority (SUSALUD) has developed an online platform, "ReSUelve tu afiliación", with the intent to solve the problems with health service access experienced by Peruvian citizens who hold health insurance policies through institutions that manage health insurance funds (IAFAS). This platform virtually articulates the main IAFAS in Peru, which receives requests from any user requiring an update on his/her affiliation status to be resolved within 24 hours. Nearly 8 months after the implementation of this platform, more than 55 thousand applications have been resolved, thus ensuring timely access to health services under the corresponding user coverage form. As a result, this platform has helped to guarantee citizens' rights to health service access in the face of infringement caused by delays in affiliation processing among the different IAFAS in Peru.
\end{abstract}

Key words: Insurance, health; Health services accessibility; Information systems; Health services coverage; Peru (source: MeSH NLM).

\section{INTRODUCCIÓN}

Desde el año 2009, Perú ha implementado cambios en el sistema de Salud. Por un lado, la Ley 29344, Ley Marco de Aseguramiento Universal en Salud (AUS) (1) define a los actores del sistema de salud en el país: el
Ministerio de Salud como ente rector; las instituciones administradoras de fondos de aseguramiento en salud (IAFAS), como financiadores de los servicios de salud; las instituciones prestadoras de servicios de salud (IPRESS), como prestadores directos de los servicios de salud, y la entonces Superintendencia Nacional de Aseguramiento

\footnotetext{
Intendencia de Investigación y Desarrollo, Superintendencia Nacional de Salud. Lima, Perú

Facultad de Ingeniería de Sistemas, Universidad Nacional Mayor de San Marcos. Lima, Perú

Facultad de Ciencias de la Salud, Universidad Peruana de Ciencias Aplicadas. Lima, Perú

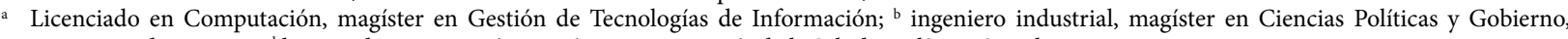
c ingeniero de sistemas; ${ }^{\mathrm{d}}$ licenciada en Nutrición, magíster en Economía de la Salud y Políticas Sociales.

Recibido: 13/05/2016 Aprobado: 15/06/2016
}

Citar como: Villegas-Ortega J, Loyola-Martínez C, Santisteban-Romero J, Manchego-Lombardi M, Lozada-Urbano M. Tecnologías de la información para resolver contingencias en la afiliación al régimen subsidiado de salud en Perú: "ReSUelve tu afiliación”. Rev Peru Med Exp Salud Publica. 2016;33(3)561-66. doi:10.17843/rpmesp.2016.333.2316 
en Salud (SUNASA) -hoy Superintendencia Nacional de Salud (SUSALUD)- como ente supervisor (2). Esta estructura y funciones son bases generales del Sistema de Salud Peruano (SSP) ${ }^{(3-4)}$.

Los aspectos financieros y de aseguramiento son clave en el SSP, y las IAFAS ocupan un lugar central en el contexto peruano. Estas instituciones pueden ser públicas o privadas; las primeras ofrecen los regímenes de aseguramiento subsidiado, semicontributivo y contributivo. Los dos primeros regímenes están a cargo del Seguro Integral de Salud (SIS) y el régimen contributivo está a cargo del Seguro Social de Salud (EsSalud), las propias de las Fuerzas Armadas y de la Policía Nacional del Perú.

Por su parte, las IAFAS privadas ofrecen seguros de salud solo en el régimen contributivo, las cuales pueden ser complementarias al régimen contributivo que ofrece EsSalud, como es el caso de las entidades prestadoras de salud (EPS). Asimismo, en el sector privado se presentan las empresas de seguros que ofertan cobertura de riesgos de salud; las IAFAS prepagas que son clínicas privadas que venden sus propios seguros, y algunas entidades empleadoras que, además de cumplir con sus contribuciones a EsSalud, administran un fondo para financiar prestaciones de salud de sus trabajadores y derechohabientes denominadas IAFAS autoseguros. Es importante manifestar que el registro completo y detallado de todas las IAFAS es administrado por SUSALUD ${ }^{(3-4)}$.

En Perú se reconoce que el aseguramiento en salud y el conocimiento certero de la situación de aseguramiento de cada peruano son cruciales. En el año 2013 el Congreso de la República delegó al Poder Ejecutivo la facultad de legislar en materia de salud y fortalecimiento del sector Salud. En ese marco se promulgó el Decreto Legislativo $1158{ }^{(5)}$, el cual establece medidas para el fortalecimiento de la SUNASA, donde se incluye el cambio de su denominación a SUSALUD con el fin de proteger los derechos en salud de los peruanos. En este mismo cuerpo legal se le otorga a SUSALUD, entre otras, la función de administrar el registro nominal de afiliados de SUSALUD (RNAS). EI RNAS constituye una base de datos nominal con validación permanente por parte del Registro Nacional de Identificación y Estado Civil (RENIEC) y se mantiene actualizado mediante el reporte constante de las IAFAS a la base de datos de SUSALUD a través de los protocolos de transferencia de archivos (en inglés: File Transfer Protocol, FTP) en donde se informa los estados actuales de los asegurados (alta, baja, cambio del estado en la afiliación del usuario); tal como está normado en la Resolución 042-2011-SUNASA/CD ${ }^{(6)}$. Consecuentemente, el RNAS es un elemento cardinal para el SSP.
A partir de lo estipulado en la Ley del AUS (1), la IAFAS SIS se ve en la necesidad de mejorar sus procedimientos de afiliación. Un paso elemental en ello, ha sido la verificación de que el solicitante no cuente con otro seguro ${ }^{(7)}$ a fin de evitar la filtración ${ }^{\left({ }^{8}\right)}$ - definida como "dar el beneficio del aseguramiento a una persona a pesar de que no es parte de la población objetivo" - tarea que era realizada sin las herramientas tecnológicas adecuadas. Es así que, dentro del contexto de las facultades otorgadas al Poder Ejecutivo, se promulgaron disposiciones para el fortalecimiento de la IAFAS SIS ${ }^{(9)}$, donde se establece que: El SIS utilizará el RNAS como única fuente de información para determinar si una persona cuenta con otro seguro de salud. En consecuencia, específicamente para el caso del SIS, el RNAS es un pilar fundamental en los procesos de aseguramiento de las personas.

Las diversas IAFAS en el Perú presentan características particulares, no solo por la distribución de frecuencia de las personas aseguradas, sino también por los distintos tipos de seguro y condiciones para el aseguramiento. De acuerdo a lo reportado por el RNAS, para abril del año 2016, el SIS cuenta con aproximadamente 16,4 millones de afiliados en el régimen subsidiado, lo que representa el $52,1 \%$ de la población del país -a manera de comparación, en México, al año 2012, el Seguro Popular subsidiado tiene afiliado al $40,8 \%$ de su población- (10) y poco más de 300 mil en el régimen semicontributivo orientado a microempresas y trabajadores independientes. El régimen subsidiado del SIS no solo afilia a pobres y pobres extremos - que para el año 2014 fueron alrededor de 6,9 millones, según el Instituto Nacional de Estadística e Informática ${ }^{(11)}$-, sino también a las denominadas poblaciones en situación de vulnerabilidad o riesgo social. Para ello, el Ministerio de Desarrollo e Inclusión Social (MIDIS), a través del sistema de focalización de hogares (SISFOH) ${ }^{(12)}$ se encarga de calificar como elegibles a la población a los programas subsidiados otorgados por el Estado. Estar registrado en el SISFOH como elegible, es un requisito para ingresar al régimen subsidiado que ofrece el SIS, a lo cual se debe sumar el hecho de no contar con otro seguro de salud.

Por otro lado, EsSalud ${ }^{(13)}$, y de manera complementaria las EPS, ofrecen el denominado seguro regular; en el cual se requiere que el usuario trabaje en una entidad empleadora. Este derecho alcanza al trabajador y sus derechohabientes legales. EsSalud, a abril de 2016, tiene aproximadamente nueve millones de afiliados regulares, y se estima que esa cifra representa el $56 \%$ del total de afiliados que deberían estar en el régimen contributivo ${ }^{(14)}$. Ello podría reflejar altos niveles de subcobertura en el seguro contributivo y de informalidad laboral en el país ${ }^{(15-16)}$. 
Por tanto, se podría afirmar que en las dos más grandes IAFAS (SIS y EsSalud) existen problemas de filtración y subcobertura, con la particularidad de que parte de la subcobertura en EsSalud termina como filtración en el SIS.

\section{DEFINICIÓN DEL PROBLEMA}

Un problema recurrente el año 2015, observado por SUSALUD, fue la presentación de quejas ante la Intendencia de Investigación y Desarrollo (IID) y la Intendencia de Protección de Derechos en Salud (IPROT) que hacían referencia a "limitaciones para acceder a servicios de salud debido a que el estado de afiliación de los solicitantes, principalmente del SIS, no se encontraba actualizado en alguna otra IAFAS". Ello ocasionaba trámites que podían durar entre una semana y varios meses; en ese tiempo, las personas debían solicitar la actualización de su estado de afiliación a la IAFAS que previamente pertenecía. Tras ello, las IAFAS analizaban cada solicitud y, de ser necesario, actualizaban el RNAS. En tanto el RNAS no estuviera actualizado, el usuario no se podía afiliar al SIS, ergo, no podía acceder a los servicios de salud correspondientes.

Para revertir esta situación, SUSALUD, a través de la IID, se planteó el reto de diseñar una plataforma en línea orientada al usuario que ordene, simplifique y resuelva los problemas de afiliación al SIS, relacionados a la desactualización del estado de la afiliación en un plazo máximo de 24 h y en forma gratuita. Hay que precisar que la solución nace como una iniciativa estratégica producto de reuniones de trabajo entre especialistas de la IID, entrevistas con algunas IAFAS involucradas y a la experiencia de la IAFAS EsSalud con la denominada acreditación contingente implementada en el año 2004, la cual buscaba resolver problemas de acceso de sus asegurados cuando su información estaba desactualizada.

Debido a que en el país se cuenta con diversos regímenes de aseguramiento, no se logró identificar publicaciones respecto a experiencias similares a "ReSUelve tu afiliación" en otros países. Aunque existen casos como Colombia que hace uso de la herramienta informática conocida como Sistema de Afiliación Transaccional, el cual gestiona la afiliación de manera integrada a todos los agentes de salud ${ }^{(17)}$.

\section{METODOLOGÍA DE LA IMPLEMENTACIÓN DE LA PLATAFORMA EN LÍNEA}

Para el desarrollo de esta iniciativa se concibieron cuatro fases:

En la Fase I, Planeamiento y Análisis de la Situación, se realizaron reuniones de trabajo y coordinación entre SUSALUD con el SIS, EsSalud y otras IAFAS, a fin de dimensionar el problema. Se concluyó que EsSalud era la IAFAS que podría presentar más dificultades. Luego, se diseñó y aprobó el procedimiento que debía ser soportado por la plataforma en línea; esta fase culminó con el compromiso de las IAFAS públicas y privadas de atender las solicitudes de verificación de la afiliación en un plazo máximo de $24 \mathrm{~h}$.

La Fase II, Desarrollo e Implementación de la Plataforma en línea, tuvo como principal fuente de información al RNAS que contiene los datos de afiliación de más de 25 millones de peruanos, todos validados con el RENIEC. También se elaboró un protocolo para la gestión de operadores de la plataforma en línea, se realizaron pruebas de operación durante un mes entre las IAFAS SIS y EsSalud. A este nivel se evaluaron resultados y se efectuaron mejoras funcionales.

En la Fase III, Capacitación y Difusión, se instruyó a los operadores que conformarían la plataforma en línea $y$, a su vez, se le dotó de identidad comunicacional denominándola "ReSUelve tu afiliación" con la finalidad de facilitar su identificación. Asimismo, se elaboró un tutorial virtual que se publicó en el portal institucional de SUSALUD, para luego difundirla a través de diversos medios de comunicación.

Finalmente, durante la Fase IV, Puesta en Marcha $y$ Operación, se descentralizó gradualmente en el ámbito nacional. Actualmente se monitorea a través de una herramienta de inteligencia de negocios (Qlikview 11, @ QlikTech International) y se evalúa su funcionalidad para identificar oportunidades de mejoras.

\section{PAUTAS DE FUNCIONAMIENTO DE LA PLATAFORMA EN LÍNEA}

El flujo de solicitudes inicia cuando un usuario requiere afiliarse al SIS en uno de sus centros de afiliación y el operador que lo atiende consulta el estado de la afiliación en el RNAS. En caso se encuentre otra afiliación activa y el usuario no esté conforme, el operador del centro de afiliación solicita la verificación a través de la plataforma en línea "ReSUelve tu afiliación". La IAFAS que recibe la solicitud, la evalúa y, si corresponde modificar el estado de la afiliación en virtud a la solicitud del usuario, actualiza directamente el RNAS a través de la plataforma en línea notificando automáticamente la atención de la solicitud. De esta manera, cuando en el centro de afiliación del SIS se vuelve a consultar al RNAS, el usuario ya no tendrá inconvenientes para su afiliación. A nivel de la plataforma en línea, este resultado exitoso a la demanda del usuario es considerado como un hit. Cuando la IAFAS se ratifica en su posición inicial y no modifica el estado de la afiliación, significa que el asegurado tiene derecho de atención en salud financiado por esa IAFAS y no puede afiliarse al SIS o requiere presentar un documento adicional para sustentar su solicitud. (Figura 1). 


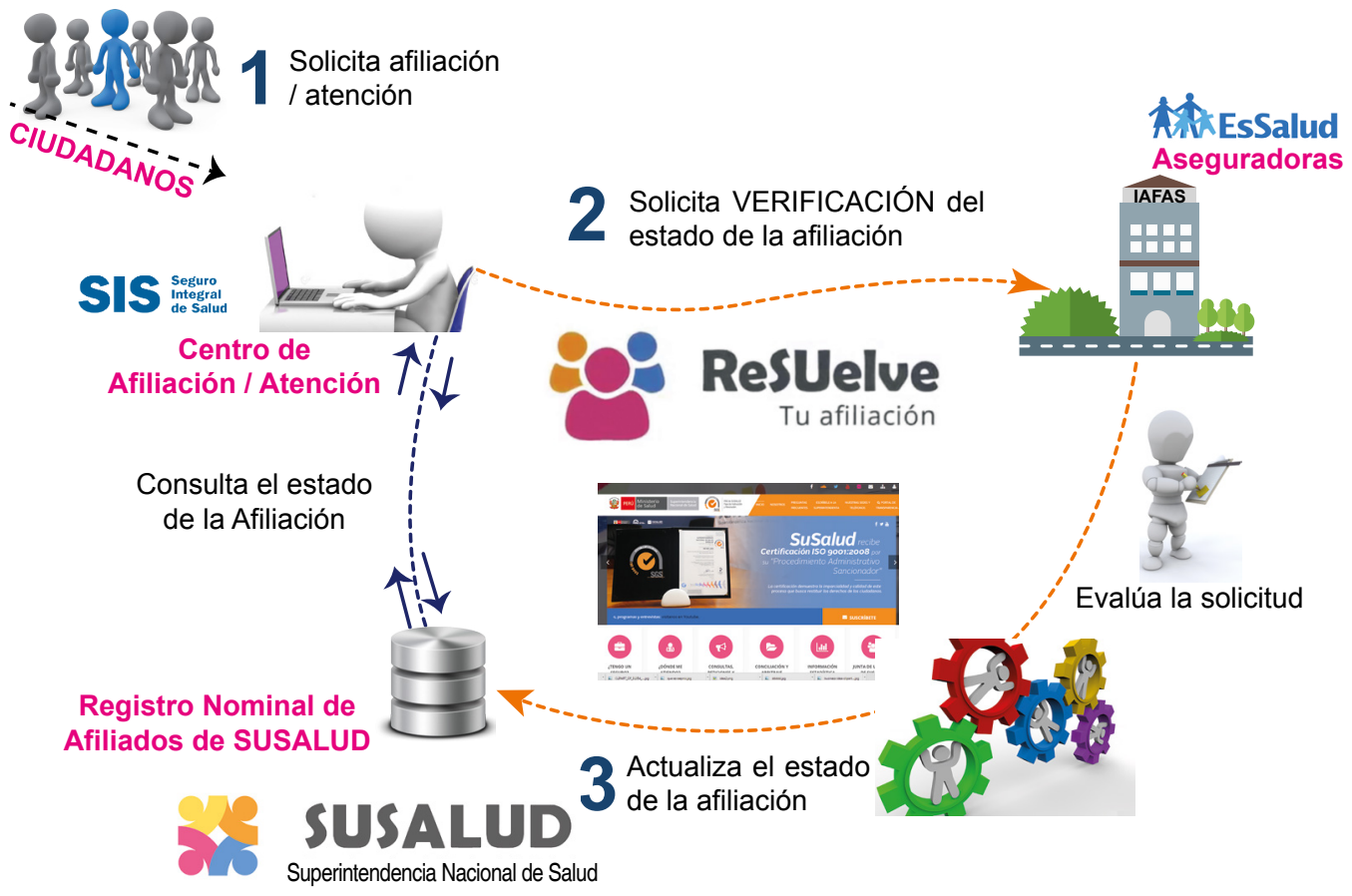

Figura 1. Flujo de solicitudes. Plataforma en línea "ReSUelve tu afiliación"

\section{RESULTADOS}

Desde el 14 de septiembre de 2015 se cuenta con una plataforma en línea conformada por 350 operadores asignados a la atención al usuario entre todas las IAFAS involucradas, incluyendo 49 dependencias del SIS que ejecutan un procedimiento simplificado apoyado en tecnologías de información y comunicación.

Hasta el mes de abril del año 2016 se han atendido 55167 solicitudes, de las cuales el $72,4 \%$ resultaron favorables para el solicitante (hits) lo que permitió la actualización de esas afiliaciones. No obstante, en el $27,6 \%$ de las solicitudes, el usuario no ha tenido la razón o debe cumplir adicionalmente con la presentación de documentación que acredite su condición laboral (Tabla 1).

En cuanto a la oportunidad de la atención, el $86,3 \%$ de las solicitudes son resueltas oportunamente, es decir, en menos de $24 \mathrm{~h}$. Cabe señalar que el $13,7 \%$ restante incluye las solicitudes registradas los fines de semana (viernes y sábados) que se atienden el lunes siguiente.

Asimismo, en la Tabla 2 se aprecia que el $96,9 \%$ de las solicitudes de verificación son dirigidas a EsSalud, lo restante ha sido requerido a otras 28 IAFAS.

\section{CONCLUSIONES Y REFLEXIONES}

La implementación de la plataforma en línea "ReSUelve tu afiliación" demuestra que es posible el despliegue de un esfuerzo colectivo e interinstitucional, principalmente de entidades estatales, en generar una red virtual que tenga como meta resolver las solicitudes del estado de afiliación de los usuarios de los servicios de salud en Perú. Esta iniciativa representa una mejora substancial en la eficiencia de los trámites administrativos relacionados al aseguramiento, con una marcada reducción de tiempo y costos tanto para el usuario como para el SSP.

Resulta importante precisar que más allá del presupuesto limitado, la experiencia ha sido posible gracias al apoyo de importantes aliados de SUSALUD: SIS, EsSalud y medios de comunicación. Instituciones que permitieron

Tabla 1. Número de solicitudes atendidas por Resuelve por resultados según mes (septiembre 2015 - abril 2016)

\begin{tabular}{lccc}
\hline Mes & $\begin{array}{c}\text { Total de } \\
\text { Solicitudes }\end{array}$ & Hits $^{*}$ & \% Hits \\
\hline Septiembre & 598 & 488 & $81,6 \%$ \\
Octubre & 2028 & 1503 & $74,1 \%$ \\
\hline Noviembre & 4897 & 3315 & $67,7 \%$ \\
\hline Diciembre & 5995 & 4083 & $68,1 \%$ \\
\hline Enero & 9235 & 6569 & $71,1 \%$ \\
\hline Febrero & 10854 & 7682 & $70,8 \%$ \\
Marzo & 12226 & 9310 & $76,1 \%$ \\
\hline Abril & 9334 & 6975 & $74,7 \%$ \\
Total & 55167 & 39925 & $72,4 \%$ \\
\hline
\end{tabular}

* Hits: Resultado favorable al solicitante. 
Tabla 2. Solicitudes atendidas por ReSUelve por meses y según IAFAS (septiembre 2015 - abril 2016)

\begin{tabular}{|c|c|c|c|c|c|c|c|c|c|}
\hline IAFAS* & Septiembre & Octubre & Noviembre & Diciembre & Enero & Febrero & Marzo & Abril & Total \\
\hline EsSalud & 527 & 1904 & 4632 & 5753 & 8892 & 10539 & 11918 & 9050 & 53215 \\
\hline SALUDPOL & 7 & 33 & 126 & 95 & 182 & 114 & 105 & 127 & 789 \\
\hline Rímac EPS & 28 & 46 & 60 & 43 & 11 & 18 & 38 & 25 & 269 \\
\hline Pacífico EPS & 5 & 7 & 19 & 22 & 40 & 59 & 39 & 27 & 218 \\
\hline FOSPEME & 0 & 1 & 4 & 29 & 38 & 37 & 30 & 15 & 154 \\
\hline Mapfre EPS & 1 & 5 & 5 & 13 & 24 & 12 & 16 & 16 & 92 \\
\hline FOSMAR & 8 & 6 & 19 & 8 & 5 & 14 & 11 & 15 & 86 \\
\hline Rímac Seguros & 0 & 0 & 4 & 6 & 6 & 19 & 23 & 13 & 71 \\
\hline Pacífico Seguros & 8 & 2 & 1 & 5 & 5 & 6 & 5 & 8 & 40 \\
\hline BNP PARIBAS CARDIF & 1 & 2 & 6 & 4 & 3 & 10 & 7 & 5 & 38 \\
\hline Clínica Good Hope & 4 & 7 & 4 & 0 & 8 & 5 & 6 & 6 & 40 \\
\hline ONCOSALUD SAC & 2 & 2 & 1 & 5 & 6 & 4 & 6 & 1 & 27 \\
\hline Positiva Seguros & 0 & 1 & 2 & 5 & 5 & 5 & 1 & 8 & 27 \\
\hline Sanitas Perú EPS & 0 & 0 & 2 & 1 & 2 & 3 & 4 & 5 & 17 \\
\hline PAMEF SEDAPAL & 0 & 1 & 3 & 1 & 2 & 2 & 4 & 3 & 16 \\
\hline FOSFAP & 2 & 5 & 0 & 1 & 0 & 0 & 1 & 4 & 13 \\
\hline Clínica San Pablo & 0 & 0 & 4 & 1 & 2 & 1 & 1 & 2 & 11 \\
\hline Mapfre Seguros & 0 & 1 & 2 & 0 & 2 & 1 & 4 & 0 & 10 \\
\hline CSALUD S.A. & 0 & 3 & 1 & 1 & 1 & 1 & 2 & 0 & 9 \\
\hline CORPAC & 0 & 0 & 0 & 0 & 0 & 0 & 2 & 2 & 4 \\
\hline Clínica Montefiori & 1 & 2 & 0 & 0 & 1 & 0 & 0 & 0 & 4 \\
\hline FESUNAT & 2 & 0 & 0 & 0 & 0 & 1 & 1 & 0 & 4 \\
\hline PAMF PETROPERÚ & 0 & 0 & 0 & 1 & 0 & 1 & 0 & 2 & 4 \\
\hline SIMA & 0 & 0 & 1 & 0 & 0 & 0 & 2 & 0 & 3 \\
\hline Clínica San Borja & 2 & 0 & 0 & 0 & 0 & 0 & 0 & 0 & 2 \\
\hline BCR del Peru & 0 & 0 & 1 & 0 & 0 & 0 & 0 & 0 & 1 \\
\hline MEDEX & 0 & 0 & 0 & 1 & 0 & 0 & 0 & 0 & 1 \\
\hline Ministerio de Comercio & 0 & 0 & 0 & 0 & 0 & 1 & 0 & 0 & 1 \\
\hline Ministerio de Economía & 0 & 0 & 0 & 0 & 0 & 1 & 0 & 0 & 1 \\
\hline Total & 598 & 2028 & 4897 & 5995 & 9235 & 10854 & 12226 & 9334 & 55167 \\
\hline
\end{tabular}

*IAFAS: Instituciones Administradoras de Fondos de Aseguramiento en Salud. SALUDPOL: Fondo de aseguramiento en salud de la Policía Nacional del Perú, EPS: Entidad prestadora de Salud, FOSPEME: IAFAS del Ejército Peruano, FOSMAR: Fondo de seguro de la Marina de Guerra del Perú, PAMEF SEDAPAL: Programa de seguro médico familiar de la empresa de Servicio de Agua y Alcantarillado de Lima, FOSFAP: IAFAS de la Fuerza Aérea del Perú, CSALUD: IAFAS "El club de la Salud" sociedad anónima, CORPAC: IAFAS de la Corporación Peruana de Aeropuertos y Aviación Comercial, FESUNAT: Fondo de empleados de la Superintendencia Nacional de Aduanas y administración tributaria, PAMF PETROPERÚ: Programa de asistencia médico familiar de la empresa Petróleos del Perú S.A., SIMA: Servicios industriales de la Marina S.A., BCR: Banco Central de Reserva.

fortalecer el trabajo y la difusión de la plataforma y aportaron con colaboradores ad-hoc para esta iniciativa.

El llevar a cabo esta experiencia ha revelado tres aspectos capitales: la frecuencia de actualización de la información de las IAFAS en el RNAS, la articulación de las IAFAS que administran dichos regímenes, y los factores externos que dificultan el acceso a la cobertura de salud.

Primero, se hizo evidente que la frecuencia de actualización respondía a un problema estructural relacionado a procesos internos a cargo de la IAFAS EsSalud, que debían ser solucionados con el fin de garantizar los derechos en salud de cada usuario. A medida que la información de las IAFAS se actualice con mayor frecuencia y oportunidad en el RNAS, se espera que las solicitudes que atiende la plataforma en línea "ReSUelve tu afiliación" debieran disminuir y, con ello, la cantidad de solicitudes de ReSUelve puede convertirse en un buen indicador de eficiencia del RNAS. En ese sentido, SUSALUD ha establecido, a través de la Resolución 081-2015-SUSALUD/S, la modalidad en línea del RNAS que permitirá a todas las IAFAS enviar la información en el momento que ocurra la novedad, en vez de la acumulación de novedades para la modalidad en lotes, como es actualmente. Hacer que esto sea posible es una tarea que aún se está desarrollando y permitirá garantizar el derecho de acceso al servicio de salud de todos los peruanos.

Segundo, respecto de la necesidad de articulación entre las IAFAS del Estado (SIS y EsSalud) que administran ambos regímenes, es necesario buscar alternativas que disminuyan trámites al asegurado. Es decir, es positiva la reducción del tiempo de más de una semana a menos 
de un día, pero sigue habiendo una necesidad de realizar un trámite ante las IAFAS por parte del usuario que ingresa y sale del empleo formal. En ese sentido, se plantea, por ejemplo, establecer un mecanismo que asegure a la IPRESS por parte de las IAFAS el pago de los servicios de salud brindados a los usuarios, independientemente de su afiliación.

Tercero, el acceso a la cobertura presenta aún varias tareas pendientes que desarrollar. Entre ellas, la informalidad, la responsabilidad de las entidades empleadoras en las declaraciones laborales y su desfase en la oportunidad del envío de información a EsSalud, las personas que cuentan con empleos eventuales, las políticas y normas de las IAFAS en cuanto a los periodos de carencia y latencia condicionadas en el momento del acceso y la actualización oportuna en el RNAS, que aunque no son tratados en este artículo, son temas prioritarios que deben convertirse en materia de investigación en la arena del aseguramiento y servicios de salud.

\section{PERSPECTIVAS}

El siguiente paso para SUSALUD es extender la plataforma virtual "ReSUelve tu afiliación" para que cualquier usuario pueda, no solo consultar su afiliación en una IAFAS (disponible en http://portales.susalud.gob. pe/web/cdi/consulta-al-registro-de-afiliados-del-aus), sino solicitar la verificación de la misma y hacer el seguimiento a su afiliación, todo ello disponible desde su computadora o equipo móvil (smartphone). De esta manera, la plataforma virtual se orientaría no solo a resolver los problemas en el momento en que el usuario requiere atenderse, sino tendría un fuerte componente preventivo.

Contribuciones de autoría: JVO, JSR y CLM participaron en la concepción del artículo; JVO, CLM, MML y MLU realizaron contribución en el diseño del artículo; CLM, JSR y MML contribuyeron con la adquisición, análisis e interpretación de datos; JVO, CLM, MLU y JSR elaboraron el borrador del artículo; JVO, CLM, MLU, JSR y MML hicieron la revisión crítica del contenido y brindaron aporte substancial. Todos los autores aprobaron la versión final. Todos los autores se hacen responsables del contenido del artículo.

Fuentes de financiamiento: Superintendencia Nacional de Salud (SUSALUD).

Conflictos de interés: al momento de la publicación los autores laboran en la Superintendencia Nacional de Salud (SUSALUD). No obstante, este documento no representa un posicionamiento oficial por parte de SUSALUD.

\section{REFERENCIAS BIBLIOGRÁFICAS}

1. Perú, Congreso de la República. Ley Marco del Aseguramiento Universal en Salud. Ley 29344. El Peruano. 8 de abril de 2009.

2. Wilson L, Velásquez A, Ponce C. La ley marco de aseguramiento universal en salud en el Perú: análisis de beneficios y sistematización del proceso desde su concepción hasta su promulgación. Rev Peru Med Exp Salud Publica. 2009;26(2):207-17.

3. Alcalde-Rabanal JE, Lazo-González O, Nigenda G. Sistema de salud de Perú. Salud Publica Mex. 2011; 53(Supl 2):s243-54.

4. Sánchez-Moreno F. El sistema nacional de salud en el Perú. Rev Peru Med Exp Salud Publica. 2014;31(4):747-53.

5. Perú, Ministerio de Salud. Decreto legislativo que dispone medidas destinadas al fortalecimiento y cambio de denominación de la Superintendencia Nacional de aseguramiento en Salud. Decreto Legislativo 1158. El Peruano. 6 de diciembre de 2013.

6. Perú, Superintendencia Nacional de Salud. Reglamento del Registro de Afiliados al Aseguramiento Universal en Salud AUS. Resolución de Superintendencia 042-2011-SUNASA/CD. El Peruano. 6 de Junio de 2011).
7. Decreto Supremo 008-2010-SA. Aprueban Reglamento de la Ley 29344, Ley Marco del Aseguramiento Universal en Salud. (El Peruano, 3 de abril, 2010).

8. Jaramillo M, Parodi S. Documento de trabajo 46. "El Seguro Escolar Gratuito y el Seguro Materno Infantil: Análisis de su incidencia e impacto sobre el acceso a los servicios de salud y sobre la equidad en el acceso". Lima: GRADE; 2004.

9. Perú, Ministerio de Salud. Decreto legislativo que aprueba disposiciones para el fortalecimiento del Seguro Integral de Salud. Decreto Legislativo 1163. El Peruano. 7 de diciembre de 2013.

10. Consejo Nacional de Evaluación de la Política de Desarrollo Social. Indicadores de acceso y uso efectivo de los servicios de salud de afiliados al Seguro Popular. México, DF: CONEVAL; 2014.

11. Instituto Nacional de Estadística e Informática. Evolución de la Pobreza Monetaria 2009-2014. Informe Técnico [Internet]. Lima: INEI; 2015 [Citado el 11 de mayo de 2016]. Disponible en: http://www.inei.gob.pe/media/ cifras_de_pobreza/informetecnico_ pobreza2014.pdf
12. Perú, Ministerio de Desarrollo e Inclusión Social. Directiva que regula la Operatividad del Sistema de Focalización de Hogares. Resolución 023-2015-MIDIS. El Peruano. 22 de enero de 2015.

13. Essalud.gob.pe [Internet]. Lima: Seguro Social de salud del Perú; 2016 [Citado el 11 de mayo 2016]. Disponible en: http://www.essalud.gob.pe

14. Casalí P, Sánchez W. Evolución de la Seguridad Social en el Perú, 2001-2012. Lima: Mimeo; 2014.

15. García N. Reformas, Crecimiento e Informalidad. Propuesta de Política. Lima: CIES; 2010.

16. Loayza N. Causas y consecuencias de la informalidad en el Perú. Estudios Económicos. 2008;15:43-64.

17. Colombia, Ministerio de Salud y Protección Social. Decreto número 2353 de 2015. Bogotá: MINSALUD; 2015.

Correspondencia: José Villegas-Ortega Dirección: Avenida Velasco Astete 1398, Santiago de Surco, Lima 33, Perú. Teléfono: (+511) 3726150 anexo 5411 Correo electrónico: jvillegas@susalud.gob.pe, rexuma@gmail.com 